\section{AB0237 ANALYSIS OF THE IMPACT OF CONCOMITANT USE OF CORTICOSTEROIDS ON THE CLINICAL OUTCOMES OF PATIENTS WITH LONG-TERM RHEUMATOID ARTHRITIS IN DIFFERENT TREATMENTS}

M.J. Carmo ${ }^{1}$, M.M. Pereira ${ }^{1}$, M.D.S. Mastella ${ }^{1}$, I.M.S. Vale ${ }^{1}$, E. Pereira ${ }^{2}$, M. K. Schmitz ${ }^{1}$, S.C.D.M.S. Fialho ${ }^{3}$, G.R.W.D. Castro ${ }^{1}$, I.A. Pereira ${ }^{1} .{ }^{1}$ Rheumatology; ${ }^{2}$ Epidemiology, University of South Santa Catarina, Palhoça; ${ }^{3}$ Rheumatology, Federal University of Santa Catarina, Florianópolis, Brazil

Objectives: To analyse the impact of the concomitant use of corticosteroids on the treatment of patients with long-term rheumatoid arthritis in different clinical outcomes.

Methods: A cross-sectional study was carried out in a sample of 100 patients with rheumatoid arthritis attended at specialised centres in rheumatology in Florianópolis. Validated instruments were used to evaluate the disease activity (DAS28 VHS, DAS-28 PCR, SDAI and CDAI), functional ability (HAQ-DI) and quality of life (SF-12 and SF-6D). Statistical analysis was performed using qui-square test (Pearson), Fisher's exact test, Student's t-test, seeking to study the association between the independent variables and the use of corticosteroids.

Results: The mean disease duration of the patients was $15.1 \pm 8.7$ years, which of, $25 \%$ used corticosteroids, with an average dose of $6.7 \pm 3.9 \mathrm{mg} /$ day. The mean of the physical component summary (PCS) of SF-12 increased significantly by 1.87 points $(47.72 \pm 3.23$ vs $45.85 \pm 2.52 p=0.013)$ in patients who used corticosteroids, on the other hand, in the mental component summary (MCS) we observed a reduction of the mean by 1.5 points $(57.91 \pm 3.68$ vs $56.41 \pm 2.98 \mathrm{p}=0.046)$ in these patients. In the association between the use of corticosteroids with ICADs, HAQ and SF-6D no differences were found independent of the synthetic and/or biological DMARD used.

Conclusions: Use of corticosteroids in different treatment strategies is not associated with an increase in the frequency of clinical remission in patients with rheumatoid arthritis. In patients using corticosteroids there was a better quality of life evaluated by the PCS, but not by the MCS.

Disclosure of Interest: None declared

DOI: 10.1136/annrheumdis-2018-eular.2073

\section{AB0238 PATIENT-CENTRED APPROACH TO MANAGEMENT OF INFLAMMATORY ARTHRITIS WAS ASSOCIATED WITH IMPROVED SATISFACTION OF CARE AND PERCEIVED TREATMENT BENEFIT}

L. Attipoe, S. Virdee, J. Greenan-Barrett, D. Shah, A. Cotton, J. Guinto, J. Wilton, C. Ciurtin. Department of Rheumatology, University College London Hospital, London, UK

Background: Recently, there has been a paradigm shift from a paternalistic doctor-patient relationship towards more holistic, patient-centred care aimed at promoting patient autonomy. However, the effect of patient-centred care on important clinical outcomes, like patient satisfaction and disability, is unknown.

Objectives: To investigate how the various aspects of patients' experiences of the care they received for their inflammatory arthritis was correlated with disease activity and health satisfaction, and use this to guide our clinical practice.

Methods: 115 questionnaires (including 51 questions, in addition to HAQ) were randomly given to patients with inflammatory arthritis who attended rheumatology outpatient clinics at University College Hospital, London between November 2014 and January 2015 (104 were returned $-90 \%$ response rate). Questions were semi-structured using a Likert scale and focused on diagnosis, symptomatology, treatment history, health status and experiences of clinic attendance. The strength of association between health satisfaction and experience of care was correlated using Spearman's correlation test $(p<0.05$ was considered statistically significant)

Results: We calculated correlations between various aspects of patients' experiences of their care with other components of the questionnaire (table 1).

Conclusions: Patients who felt that they experienced a more patient-centred approach (through greater involvement in clinical decisions, emotional support and ease of contact with specialists) reported greater satisfaction and regarded their treatment as more efficacious. This suggests that clinicians who go beyond their role of medically managing inflammatory arthritis to provide more holistic care may improve patient-orientated health outcomes.

Patients in older age groups find it easier to contact the department, perhaps because they have more free time to make and receive calls. As ease of contact is a strong predictor of overall patient satisfaction, employing ideas to increase accessibility for younger age groups, such as improved access beyond working hours may improve satisfaction.

The positive correlation of $\mathrm{HAQ}$ with employment status and age is expected, as $\mathrm{HAQ}$ is the current gold standard measure of functional status. This reaffirms its accuracy in clinical practice as a measure of a functional ability.

Further research into comparing patients' experience of their care with objective measures of disease activity is needed.
Abstract AB0238 - Table 1

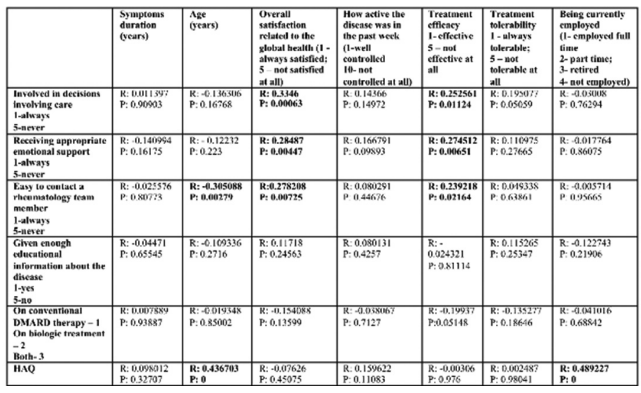

Disclosure of Interest: None declared

DOI: 10.1136/annrheumdis-2018-eular.2762

\section{AB0239 DEVELOPMENT OF A NOVEL ,MOLECULAR DISEASE ACTIVITY" TOOL COMPOSED OF 8 SERUM BIOMARKERS FOR MONITORING RHEUMATOID ARTHRITIS}

J. Hurnakova ${ }^{1}$, M. Komarc ${ }^{2}$, R. Horvath ${ }^{3,4,5}$, H. Hulejova ${ }^{1}$, J. Zavada ${ }^{1}$, L. Palova Jelinkova $^{4,5}$, K. Danova ${ }^{4,5}$, P. Hanova ${ }^{1}$, H. Mann ${ }^{1}$, J. Vencovsky ${ }^{1}$, K. Pavelka ${ }^{1}$,

R. Spisek ${ }^{4,5}$, L. Senolt ${ }^{1}{ }^{1}$ Institute of Rheumatology, 1 st Faculty of Medicine, Charles University, Prague, Czech Republic; ${ }^{2}$ Dpt. of Methodology, Faculty of Physical Education and Sport, Charles University, Prague, Czech Republic; ${ }^{3} D p t$. of Pediatric and Adult Rheumatology, University Hospital Motol, Prague, Czech Republic; ${ }^{4}$ Dpt. of Immunology, University Hospital Motol and 2nd Faculty of Medicine, Charles University, Prague, Czech Republic; ${ }^{5}$ Sotio a.s., Prague, Czech Republic

Background: Accurate disease activity measurement is a key component of rheumatoid arthritis (RA) management. In 2012, multi-biomarker disease activity (MBDA) test using 12 serum proteins was presented ${ }^{1}$.

Objectives: To develop a novel test for measuring inflammatory activity in RA. Methods: Serum samples were obtained from 82 patients with RA (60 females, median disease duration 4.2 years, median age 55.2 years, RF positivity $60.3 \%$, ACPA positivity $69.7 \%$ ). A detailed stepwise analysis of 20 candidate biomarkers selected from the literature search (IL1 $\beta$, IL-6, IL-7, IL-8, IL-12p70, IL-17A, IL-22, IL-33, IL-34, IFN $\gamma$, VEGF, YKL-40, CXCL-13, MMP-3, resistin, visfatin, leptin, adiponectin, calprotectin and CRP) was performed. Methods of factor analysis (a statistical method used to describe variability among observed, correlated variables in terms of a potentially lower number of unobserved variables called factors) were used in order to develop a tool composed of different serum markers that would optimally reflect disease activity in RA. Spearman correlation index was used to explore associations between newly designed tool and clinical as well ultrasound parameters (German US7 score) of disease activity.

Results: We have developed a "Molecular Disease Activity" test covering underlying pathophysiological processes composed of 8 serum markers: calprotectin CRP, IL-6, MMP-3, VEGF, resistin, IL-22 and IL-7 that optimally reflected inflammatory activity in RA. This model was significantly associated with clinical (DAS28-ESR, DAS29-CRP, CDAI, SDAI) and ultrasound variables (PD syn score, GS syn score) of disease activity in RA, as shown in table 1.

Abstract AB0239 - Table 1. Cross-sectional correlations between "Molecular Disease Activity" tool composed of 8 biomarkers and clinical and ultrasound parameters of disease activity

\begin{tabular}{lcc}
\hline Parameters & $\begin{array}{c}\text { Molecular disease activity } \\
\text { (factor score) }\end{array}$ & $p$ (significance) \\
\hline DAS28-ESR & $0.664^{\star *}$ & $<0.001$ \\
DAS28-CRP & $0.666^{* *}$ & $<0.001$ \\
SDAI & $0.731^{* *}$ & $<0.001$ \\
CDAI & $0.669^{* *}$ & $<0.001$ \\
SJC & $0.546^{* *}$ & $<0.001$ \\
GS syn score & $0.657^{* *}$ & $<0.001$ \\
PD syn score & $0.701^{* *}$ & $<0.001$ \\
${ }^{* *}$ Correlation is significant at $\leq 0.01$ & &
\end{tabular}

CDAI, Clinical Disease Activity Index; DAS28-CRP, Disease Activity Score with C-reactive protein; DAS28-ESR, Disease Activity Score with erythrocyte sedimentation rate; GS syn, Grey Scale synovitis; PD syn, Power Doppler synovitis; SDAI, Simplified Disease Activity Index; SJC, swollen joint count

Conclusions: We believe that this newly designed test based on 8 serum markers may contribute to more accurate measurement of inflammatory activity in 